\title{
GENES DIFFERENTIALLY EXPRESSED BY Mycobacterium tuberculosis AFTER EXPOSURE TO RUTHENIUM PHOSPHINIC COMPOUND AND ISONIAZID
}

\author{
LEITE G.G.S. ${ }^{1}$, BAEZA L.C. ${ }^{2}$ BATISTA A.A. ${ }^{3}$, BARBOSA M.I.F. ${ }^{3}$, PAVAN F.R. ${ }^{4}$, LEITE C.Q.F. ${ }^{4}$, SILVA J.L. ${ }^{5}$, \\ HIRATA R.D.C. ${ }^{5}$, HIRATA M.H. ${ }^{5}$ AND CARDOSO R.F. ${ }^{*}$
}

1Postgraduate Program in Health Science, State University of Maringá, 87020-900, Maringá, Paraná, Brazil. 2Department of Clinical Analysis and Biomedicine, State University of Maringá, 87020-900, Maringá, Paraná, Brazil.

3Department of Chemistry, Federal University of São Carlos, 13561-901, São Carlos, São Paulo, Brazil.

4School of Pharmaceutical Science, São Paulo State University, 14800-901, Araraquara, São Paulo, Brazil.

5 Faculty of Pharmaceutical Sciences, University of São Paulo, 05508-900, São Paulo, Brazil.

${ }^{*}$ Corresponding Author: Email- rfressatticardoso@gmail.com

Received: February 05, 2013; Accepted: February 19, 2013

\begin{abstract}
-
Background- The evaluation of the effects of new compounds and nonconventional anti-tuberculous drugs have grown and become increasingly more popular in recent years. Studies have shown anti-tuberculous activity for Ruthenium complexes, including organometallic compounds containing phosphine ligands such as picolinic acid generating great expectations and hopes.

Methods- The Representational Difference Analysis (RDA) was applied in order to gain insight about differences in expression of Mycobacterium tuberculosis $\mathrm{H}_{37} \mathrm{Rv}$ exposed to [Ru(dppb)(pic)(bypy)]PF6 (SCAR1) and isoniazid (INH). Total RNA was extracted from the bacillus not exposed and exposed to SCAR1 and INH separately at concentration of MIC for 12 hours at $35^{\circ} \mathrm{C}$. RDA was carried out and differentially expressed products were sequenced.

Results- RDA-sequencing identified, for both compounds, orthologs that encode hypothetical and predict proteins. One related cell wall synthesis gene, identified by RDA, and genes related to INH target as inhA, katG and ahpC had their expression confirmed and quantified by real -time PCR. The gene encoding the cell wall associated hydrolase was induced 4.627 and 1.189, inhA 0.983 and 1.027, katG 1.111 and 1.345 and $a h p C 1.063$ and 1.039 fold after exposure to SCAR1 and INH respectively, compared to not exposed growth.

Conclusion- The RDA brings, for the first time, directions to study related genes with metabolic pathways of SCAR1. RDA and Real-Time PCR highlight the idea that one of the SCAR1 interaction, in $M$ tuberculosis may be in the cell wall biosynthesis considering the differential expression of a cell wall hydrolase and warrants further investigation.
\end{abstract}

Keywords- Mycobacterium tuberculosis, Isoniazid, Ruthenium, cDNA-RDA.

Citation: Leite G.G.S., et al. (2013) Genes Differentially Expressed by Mycobacterium tuberculosis after Exposure to Ruthenium Phosphinic Compound and Isoniazid. International Journal of Microbiology Research, ISSN: 0975-5276 \& E-ISSN: 0975-9174, Volume 5, Issue 1, pp.-357362. DOI : 10.9735/0975-5276.5.1.357-362

Copyright: Copyright@2013 Leite G.G.S., et al. This is an open-access article distributed under the terms of the Creative Commons Attribution License, which permits unrestricted use, distribution and reproduction in any medium, provided the original author and source are credited.

\section{Introduction}

Tuberculosis (TB) is an infectious disease that has been known for centuries. Its development and evolution are dependent on factors such as human settlements, malnutrition and impaired immune response, the latter being a factor related mainly with HIV infection, which has increased the incidence of TB in HIV-positive people [1]. In 2011, almost 9 million of new cases (125/100.000 population) of TB and approximately 1.4 million TB deaths worldwide $(990.000$ among human immunodeficiency virus (HIV) negative people and 430.000 HIV-associated TB deaths) [2].

An incomplete or inadequate treatment led to the emergence of resistant Mycobacterium tuberculosis to antituberculous (anti-TB) drugs. Studies have shown anti-TB activity by several classes of nonconventional compounds generating great expectations and hopes $[3,4]$. The evaluation of the effects of new compounds and nonconventional anti-TB drugs have grown and become increasingly more popular in recent years, mainly by the emergence of multidrug resistant TB (MDR-TB) and extensively-drug resistant TB (XDR-TB) [5-7].

Ruthenium complexes, including organometallic compounds containing phosphine ligands such as picolinic acid, have shown to be active against $M$. tuberculosis [8-11] and a number of other bacteria [12]. Nascimento et al. [9] and Pavan et al. [10,11] synthesized ruthenium complexes containing phosphines, diimines and picolinic 
acid such as ligands. These complexes' library received the acronym "SCAR" and their anti-M. tuberculosis activity, cytotoxicity and intracellular activities were investigated [9-11]. In these cases the Minimal Inhibitory Concentration (MIC) of compounds were comparable to or better than MICs of first and second line anti-TB drugs and even 100 times more active than their free ligands [9-11].

Representational Difference Analysis (RDA) is a powerful and sensitive tool for the identification of differentially expressed genes in two different cDNA populations. This technique allows detection of changes in mRNA expression by selective enrichment without any prior knowledge of the gene in question [13].

Given the knowledge of TB worldwide, the emergence of potential anti-TB compounds, along with the need for knowledge of the metabolic pathways involved in their action, this study aimed to identify transcripts in M. tuberculosis after exposure to [Ru(dppb)(pic)(bipy)] PF6 (SCAR1) and isoniazid (INH), using for the first time, as we know, the RDA.

\section{Materials and Methods \\ Ruthenium Compound}

The $[R u(d p p b)($ pic)(bipy)]PF6 ([dppb = 1,4-bis(diphenylphosphino) butane; pic = picolinic acid; bipy $=2,2^{\prime}$-bipyridine $]$ ) compound (SCAR1) used in the present study was synthesized in the Department of Chemistry, Federal University of São Carlos, Brazil according to a procedure described in the literature [12] and characterized by analytical techniques such as elemental analysis $(\mathrm{C}, \mathrm{N}, \mathrm{H}, \mathrm{S})$, cyclic voltammetry and magnetic nuclear resonance.

\section{Strain and Culture Conditions}

M. tuberculosis $\mathrm{H}_{37} \mathrm{Rv}$ ATCC 27294 mid-log phase culture in Middlebrook 7H9 medium (Difco, Detroid, MI, USA) supplemented with OADC (BBLTM - Becton Dickinson Microbiology Systems, Sparks, MD, USA) were used for experiments with SCAR1 and INH. The M. tuberculosis culture, referred as tester, was previously standardized to optical density at $0.2-0.4 \mathrm{OD}_{600}$. After, $0.91 \mu \mathrm{g} / \mathrm{ml}$ of SCAR1, according to MIC determined by REMA [14], was added to the mycobacterial growth and incubated for 12 hours at $35^{\circ} \mathrm{C}$. The $\mathrm{M}$. tuberculosis culture, referred as driver, was cultured in OADCsupplemented Middlebrook $7 \mathrm{H} 9$ medium at $35^{\circ} \mathrm{C}$. As a control of differentially expressed genes, a reverse experiment was performed where driver and tester cultures were exposed to $0.91 \mu \mathrm{g} / \mathrm{ml}$ of SCAR1 and not exposed in the same conditions as above, respectively.

Analysis with INH was carried out with the same protocol for SCAR1 using $0.03 \mu \mathrm{g} / \mathrm{ml}$ of INH (Sigma, St. Louis, MO, USA), according to MIC previously determined for $M$. tuberculosis $\mathrm{H}_{37} \mathrm{Rv}$ ATCC 27294 by REMA [14]. A reverse experiment was conducted for INH in which the driver RNA was extracted from M. tuberculosis growth added of $\mathrm{INH}$ and the tester RNA from $M$. tuberculosis growth in OADC-supplemented Middlebrook $7 \mathrm{H} 9$ medium. The concentration and time of exposure to both compounds were based in MIC and generation time of M. tuberculosis.

\section{RNA Isolation and cDNA Synthesis}

Total RNA was extracted and purified from $M$. tuberculosis growth for each experimental condition by highly denaturing guanidinethiocyanate using RNeasy Mini Kit (Qiagen Biotechnology, USA) according to manufacturer's instructions. The extracted RNA was then treated with DNAse I (Invitrogen Life Technologies, CA, USA) and agarose gel was carried out to verify their integrity and quality.
The RNA quantification and purity were carried out by Qubit ${ }^{\circledR}$ fluorometer (Invitrogen, USA) in 260/230 and 260/280 nm respectively. First-strand cDNA was synthesized with RT Superscript III (Invitrogen Life Technologies, CA, USA) and $100 \mathrm{nM}$ of primer 5'AAGCAGTGGTATCAACGCAGAGTACGCGGG 3' using $1 \mu \mathrm{g}$ of total RNA. The first strand was used as template to synthesize the second CDNA strand by using Random Primers (Invitrogen Life Technologies, CA, USA) according to manufacturer's instructions.

\section{Subtractive Hybridization and Generation of Subtracted Librar- ies}

The cDNA were digested with Sau3AI (Promega Corporation, Madison, WI, USA). A subtracted CDNA library was constructed using driver cDNA synthesized from RNA of $M$. tuberculosis cultured in OADC-supplemented Middlebrook 7H9 medium and tester cDNA from RNA extracted from M. tuberculosis exposed to SCAR1 and INH. The products were purified using QIAquick PCR Purification Kit (Qiagen Biotechnology, USA). The tester-digested cDNA was bonded to adapters RBam24 (5'AGCACTCTCCAGCCTCTCTCA CCGAG3') and RBam12 (5'GATCCTCGGTGA3'). To generate the differential products, tester and driver cDNAs were mixed, hybridized at $67^{\circ} \mathrm{C}$ for 18 hours and amplified with RBam24 primer. A second round was carried out with the adapters JBam24 (5'ACCGACGTCGACTATCCATGAACG3') and JBam12 (5'GATC CGTTCATG3'). Two successive rounds of subtractions and amplifications using hybridization tester-driver ratios of 1:10 and 1:100, were performed to generate second differential products and purified using QIAquick PCR Purification Kit (Qiagen Biotechnology, USA) $[13,15]$. The purified cDNAs products were cloned into the pGEM-T-Easy vector (Promega Corporation, Madison, WI, USA). Escherichia coli XL1 Blue competent cells were transformed with the ligation products. Plasmid DNA was extracted from selected colonies using standard protocols [16]. In order to generate the expressed sequence tags (EST), single-pass, 5 '-end sequencing of cDNAs by a standard fluorescence-labeling dye-terminator protocols with T7 flanking vector primer was performed. Samples were loaded onto a MegaBACE 1000 DNA sequencer (GE Healthcare Life Science, USA) for automated sequence analysis.

\section{Expressed Sequence Tag (EST) and Differential Expression Analysis}

ESTs were pre-processed using the Phred and Crossmatch (http:// www.genome.washington.edu/UWGC/analysstools/Swat.cfm) programs. Sequences with at least 100 nucleotides and Phred quality greater than or equal to 20 were considered for further analysis. ESTs were screened for vector sequences against the UniVec data, and assembled with the CAP3 program [17].

The filtered sequences were compared against the GenBank (http:// www.ncbi.nlm.nih.gov) non-redundant (nr) database from the National Center for Biotechnology Information (NCBI) using the BLASTX algorithm with an E-value cut-off at $10^{-5}$. If the EST sequences did not match any database sequences, the BLASTN algorithm was used (http://www.ncbi.nlm.nih.gov/BLAST/) [18]. ESTs were grouped in clusters, represented by contigs and singlets.

\section{Real-time PCR Analysis of Representative Regulated Genes in M. tuberculosis}

To confirm and estimate the relative transcript levels of the differentially expressed products after exposure to SCAR1 and INH, realtime PCR was performed. The cell wall-associated hydrolase transcript, detected by RDA, with high redundancy as 179 and 77 were 
chosen for analysis. Since INH was used as control for analysis of gene expression, genes whose products are likely involved in processes related with action and toxic effects of this drug, such as katG, inhA and ahpC, were selected too.

Real-time PCR were performed in two PCR repeats using $1 \mu$ of cDNA in $10 \mu$ of reaction mixture containing $400 \mathrm{nM}$ of each primer (Promega Corporation, Madison, WI, USA) listed in [Table-1] and 5 $\mu \mathrm{l}$ of SYBR green ROX mixture (Applied Biosystems by Life Technologies, Foster City, CA, USA) according to manufacturer's instruction. The amplification was carried out in samples of CDNA from M. tuberculosis exposed to SCAR1 and INH in a 7500 Fast Real-Time PCR (Applied Biosystems by Life Technologies, Foster City, CA, USA), using the reaction program: $50^{\circ} \mathrm{C}$ for $2 \mathrm{~min}, 95^{\circ} \mathrm{C}$ for $10 \mathrm{~min}$, and 45 cycles of $95^{\circ} \mathrm{C}$ for $15 \mathrm{~s}$ and annealing and synthesis at $60^{\circ} \mathrm{C}$ for $1 \mathrm{~min}$. Melting-curve analysis was performed to confirm the signal corresponded to a single PCR product. The cycle threshold values for the duplicate PCRs for each RNA sample were averaged, and then $2^{-\Delta \Delta C T}$ values were calculated [19]. The $16 \mathrm{~S}$ RNA (rrs) gene was used for normalization of all reaction.

Table 1- Primers used in Real time PCR analysis.

\begin{tabular}{|c|c|c|}
\hline Primers sequences & a Access $\mathrm{N}^{\circ}$ & Gene \\
\hline $\begin{array}{l}\text { Fw 5' CTCTCACGAGTCCCCACCAT 3' } \\
\text { Rv 5' ACGAGCGCAACCCTTGTC 3' }\end{array}$ & b ZP_06529677 & $\begin{array}{c}\text { cell wall-associated } \\
\text { Hydrolase } \\
\text { [Streptomyces sp.] }\end{array}$ \\
\hline $\begin{array}{l}\text { Fw 5' ATGTGTCCAAGGGCATCCA 3' } \\
\text { Rv 5' CCTTGGCCATCGAAGCAT 3' }\end{array}$ & BX842576.1 & inhA \\
\hline $\begin{array}{l}\text { Fw 5' GCCGGCGAAAACAATCAG 3' } \\
\text { Rv 5' GTGGCCGGTCAAGAAGAAGTAC 3' }\end{array}$ & BX842578.1 & katG \\
\hline $\begin{array}{l}\text { Fw 5' CGTTACCCTTCCCGATGCT 3' } \\
\text { Rv 5' CTGCGGCTTGGCTGAGTT 3' }\end{array}$ & BX842579.1 & ahpC \\
\hline $\begin{array}{l}\text { Fw 5' AGCGTGATTCCGATGAGGTTT 3' } \\
\text { Rv 5' AACGATGCCGTCGTCCAA 3' }\end{array}$ & BX842581.1 & $16 \mathrm{~S}$ (rrs) \\
\hline
\end{tabular}

aAccession number at GenBank (http://www.ncbi.nlm.nih.gov)

${ }^{b}$ Accession number (GI) at GenBank protein database (http:// www.ncbi.nlm.nih.gov)

A negative control was carried out with all reagents except $M$. tuberculosis cDNA. DNA contamination was checked by real-time PCR of RNA not treated with reverse transcriptase. After 45 rounds of amplification, no PCR products were detected in either reaction. Data from biological duplicate were analyzed by two-way ANOVA, Bonferroni's test.

\section{Results}

\section{Identification of $M$. tuberculosis Genes with Differential Ex- pression}

RDA was performed on the bacillus growth exposed to SCAR1 and INH (testers) at concentrations of MIC for 12 hours at $35^{\circ} \mathrm{C}$ and in absence of these compounds separately (driver). Different patterns of cDNA fragments was observed in comparing the testers with driver after two rounds of subtractive hybridization in RDA, indicating the presence of differentially expressed genes [Fig-1].

A total of 276 and 255 sequences from cultures exposed to SCAR1 and INH respectively were successfully sequenced [Table-2] and [Table-3]. BLASTX program analysis for SCAR1 showed a total of 9 ESTs. From these, 3 corresponded to known proteins, while 6 corresponded to proteins of unknown function, with no matches in databases. In addition, from the unknown proteins, 5 of the ESTs had not been described in $M$. tuberculosis. For INH analysis, we found a total of 8 different ESTs. From these, two corresponded to known proteins, while 6 corresponded to proteins of unknown function, with no matches in databases. From the unknown proteins, 5 of the ESTs had not been described in M. tuberculosis. The other ESTs were not studied because their low redundancy.

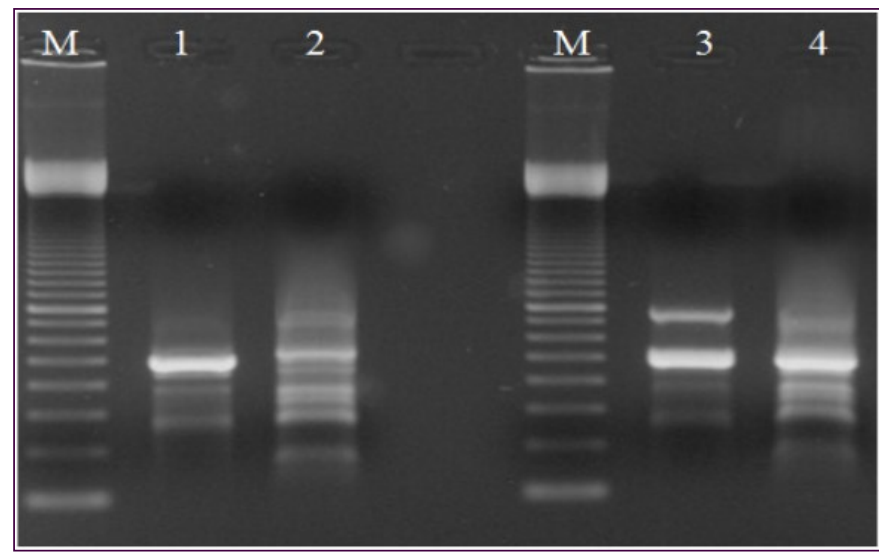

Fig. 1- Representational Difference Analysis (RDA) products analysis by gel electrophoresis. Lanes 1 and 3: Products of the first and second rounds of subtraction, performed by using as tester the cDNA obtained from RNA of Mycobacterium tuberculosis exposed to INH and SCAR1, respectively. Lanes 2 and 4: Products of the first and second rounds of subtraction, performed by using as tester the cDNA obtained from RNA of M. tuberculosis growth without INH and SCAR1, respectively. M: molecular marker 100 bp (Invitrogen, CA, USA).

\section{Quantitative Analysis of Genes by Real-Time PCR}

[Fig-2] shows quantification of the transcript levels of the most differentially expressed and the randomly selected genes in $M$. tuberculosis exposed to SCAR1 and INH at concentration of MIC for 12 hours at $35^{\circ} \mathrm{C}$. Among the genes evaluated in M. tuberculosis exposed to SCAR1 and INH, the ones encoding the cell wall associated hydrolase was induced 4.627 and 1.189 fold, inhA 0.983 and 1.027 fold, katG 1.111 and 1.345 fold and ahpC 1.063 and 1.039 fold compared to not exposed bacillus, respectively.

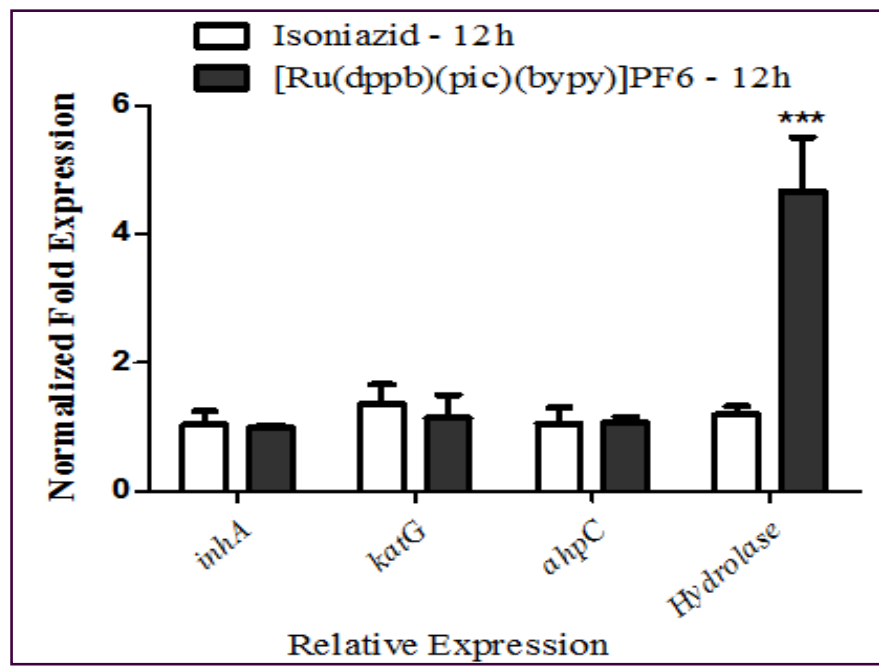

Fig. 2- Real Time PCR of the transcript levels differentially expressed inhA, katG, ahpC and cell wall associated hydrolase genes in Mycobacterium tuberculosis exposed to SCAR1 and INH for 12 hours at $35^{\circ} \mathrm{C}$. The values represent the levels of expression of genes normalized to the $M$. tuberculosis control growth in the absence of SCAR1 and INH. 
Table 2- Characterized and unknown proteins with high abundance, detected by Representational Difference Analysis (RDA), in Mycobacterium tuberculosis H37Rv exposed to 1 X MIC SCAR1 for 12 hours at $35^{\circ} \mathrm{C}$.

\begin{tabular}{|c|c|c|c|}
\hline BlastX & e-value & Redundancy & a Access $\mathbf{N}^{\circ}$ \\
\hline cell wall-associated hydrolase [Streptomyces sp.] ${ }^{b}$ & $5 e-37$ & 179 & ref|ZP_06708251.1| \\
\hline glycine cleavage system protein $\mathrm{H}$ [Hydrogenivirga sp.] & $7 e-15$ & 62 & ref|ZP_02176878.1| \\
\hline pG1 protein [Lactobacillus jensenii] & 7e-07 & 3 & ref|ZP_04645459.1| \\
\hline hypothetical protein COLAER_01671 [Collinsella aerofaciens] & $2 e-39$ & 24 & ref|ZP_01772659.1| \\
\hline LOW QUALITY PROTEIN: predicted protein [Mycobacterium tuberculosis T85] & $4 e-27$ & 4 & ref|ZP_06516795.1| \\
\hline conserved hypothetical protein [Streptomyces roseosporus] & $4 e-09$ & 1 & ref|ZP_06583083.1| \\
\hline conserved hypothetical protein [Streptomyces albus] & $1 e-12$ & 1 & ref|ZP_06593897.1| \\
\hline hypothetical protein BIFADO_00015 [Bifidobacterium adolescentis] & $9 e-07$ & 1 & ref|ZP_02027619.1| \\
\hline \multirow[t]{2}{*}{ hypothetical protein FAEPRAM212_00169 [Faecalibacterium prausnitzii] } & $8 e-08$ & 1 & ref|ZP_02089938.1| \\
\hline & Total & 276 & \\
\hline
\end{tabular}

a Accession number at GenBank (http://www.ncbi.nlm.nih.gov)

b Validated up-regulated transcript

Table 3- Characterized and unknown proteins with high abundance, detected by Representational Difference Analysis (RDA), in Mycobacterium tuberculosis $\mathrm{H}_{37} \mathrm{Rv}$ exposed to $1 \mathrm{X}$ MIC INH for 12 hours at $35^{\circ} \mathrm{C}$.

\begin{tabular}{|lccc|}
\hline BlastX & e-value & Redundancy & a Access N $^{\circ}$ \\
\hline cell wall-associated hydrolase [Streptomyces sp.] & $5 \mathrm{e}-37$ & 77 & ref|ZP_06708251.1| \\
pG1 protein [Lactobacillus jenseni] & $7 \mathrm{e}-07$ & 20 & ref|ZP_04645459.1| \\
conserved hypothetical protein [Mycobacterium tuberculosis] & $4 \mathrm{e}-43$ & 104 & ref|ZP_06512772.1| \\
hypothetical protein CE1543 [Corynebacterium efficiens] & $5 \mathrm{e}-11$ & 9 & ref|NP_738153.1| \\
hypothetical protein AYWB_261 [Aster yellows witches'-broom phytoplasma] & $6 \mathrm{e}-34$ & 39 & ref|YP_456457.1| \\
hypothetical protein CLOL250_03003 [Clostridium sp.] & $3 \mathrm{e}-13$ & 2 & ref|ZP_02076215.1| \\
hypothetical protein CE1543 [Corynebacterium efficiens] & $5 \mathrm{e}-11$ & 2 & ref|NP_738153.1| \\
hypothetical protein ACTODO_00001 [Actinomyces odontolyticus] & $3 \mathrm{e}-15$ & 2 & ref|ZP_02043164.1| \\
& TOTAL & 255 & \\
\hline
\end{tabular}

${ }^{a}$ Accession number at GenBank (http://www.ncbi.nlm.nih.gov).

\section{Discussion}

The increasing incidence of resistance to drugs used for TB therapy and co-infection with HIV, have contributed to the worsening situation of TB worldwide, mainly in developed countries, which were detected outbreaks of resistant disease [2]. Ruthenium complexes showed good activity against $M$. tuberculosis $[3,9,10]$. However, there was still no knowledge on how that compounds act in mycobacteria in their molecular basis. Even with the $\mathrm{INH}$, which is a drug used for a long time in TB treatment, there was no complete knowledge of its action and resistance in M. tuberculosis [20].

The RDA was applied in order to gain insight about differences in expression of $M$. tuberculosis exposed and not exposed to SCAR1. We used real-time PCR to confirm and quantify the differential expression of a related hydrolase of cell wall biosynthesis gene, detected by RDA in M. tuberculosis growth exposed to SCAR1 as in $\mathrm{INH}$. Three genes, which have been characterized previously as transcriptional signature of INH exposure, were selected as control of the analysis.

RDA was described mainly in eukaryotes and few studies were applied in prokaryotes [21,22] and so far, of which we have knowledge, none has been described in M. tuberculosis. This makes the application of this methodology of interest to show a first direction to conduct understanding of the action of new compounds, which there are no idea about their target in the bacillus.

Regarding the differentially expressed genes in M. tuberculosis, in answer to exposure to SCAR1, at concentration of MIC value, for 12 hours, we observed by RDA some transcripts that are related to hypothetical and predicted proteins. Also, we observed transcripts which encode proteins already characterized, with high redundancy, in particular an EST that encodes a cell wall hydrolase of Streptomyces spp. By real-time PCR, we observed overexpression (4.6 fold) of the gene encoding this protein in $M$. tuberculosis growth exposed to SCAR1, when compared to $M$. tuberculosis growth not exposed to this compound $(P<0.001)$. Haiser et al. [23] described a related hydrolase of cell wall biosynthesis in Streptomyces coelicolor, indicating the possibility of this class of enzyme be present in Mycobacteriaceae, as observed in our study.

Although little is known about the hydrolases in Mycobacteriaceae some authors $[24,25]$ have shown that they may play a role in the late stages of cell division, possibly during re-growth from a stressed state. Additionally, Chauhan et al. [24] had proposed previously that the exposure of M. tuberculosis to a variety of DNA damaging agents or certain substances leads to cell division blockage and up regulation of a protein that exhibits cell wall hydrolysis activity, which target the nascent petidoglican synthesis or it may be part of the SOS response in the DNA repair system. Considering the above findings, the overexpression of that related hydrolase of cell wall biosynthesis in SCAR1 exposed $M$. tuberculosis growth warrants further investigation. No significant changes were observed in the $\operatorname{inh} A$, katG and $a h p C$ genes expression compared to the non exposed bacillus to SCAR1.

In relation to differentially expressed genes in $M$. tuberculosis exposed to INH for 12 hours, we observed, by RDA, a higher amount of transcripts which encode some proteins not yet characterized. In the analysis performed by real-time PCR, we observed no significant changes in all studied transcript in $M$. tuberculosis growth exposed to INH compared to not exposed growth.

As we know, INH, which is a pro-drug, needs to be activated by the catalase-peroxidase enzyme (KatG), encoded by the katG, leading to the formation mainly of isonicotinic acid, among others reactive radicals. The main INH target is the inhibition of mycolic acid biosynthesis, a major constituent of the mycobacterial cell wall. For this the isonicotinic acid reacts with NAD to form the INH-NAD, inhibiting the enzyme enoyl ACP reductase $(\operatorname{Inh} A)$, encoded by inhA, then 
leading to inhibition of mycolic acid synthesis, and cell wall damage $[26,27]$. Based on this, the two above genes, implicated as molecular target for INH, and ahpC -which transcript, the alkyl hydroperoxide reductase $(\mathrm{AhpC})$ helps detoxify the cell by reducing specific classes of reactive oxygen species-, were chosen as internal control of the study.

In our study no change in inhA, katG and ahpC as the related hydrolase of cell wall biosynthesis transcripts, by INH exposure, was observed. The inhA and ahpC results corroborates with Wilson et al [28] findings, where no transcriptional changes was observed after INH treatment even using a much higher concentration of INH (1 $\mu \mathrm{g} / \mathrm{mL})$ than used in our study $(0.03 \mu \mathrm{g} / \mathrm{mL})$.

On the other side, Betts et al. [29] exposing $M$ tuberculosis $\mathrm{H}_{37} \mathrm{Rv}$ (NCTC 7416) to INH, at the same concentration used by Wilson et al. [28] observed no transcriptional changes in inhA, like our results, but ahpC was up-regulated. Waddell et al [30] and Karakousis et al. [31] observed that log-phase M. tuberculosis $\mathrm{H}_{37} \mathrm{Rv}$ exposed to INH showed significant induction of $a h p C$ among others genes. A plausible explanation for this difference in findings with ahpC expression, including our results, may be related to the concentration and exposure time of the bacillus to INH once the product of this gene is involved in processes associated with the toxic effects caused by $\mathrm{INH}$. Both, Wilson et al [28] as Karakousis et al. [31] used the same INH concentration $(1 \mu \mathrm{g} / \mathrm{mL})$ but the time of exposition is not specified by the first and 6 hours was used by the second prior to measuring expression levels. In our study, we used the concentration of MIC to expose the bacterial growth to both compounds once we intended to observe their primary effect on the bacillus and not toxic and side effects.

The time of exposure and concentration of determined compound are critical point in expression studies. A long exposure time and different concentration may reflect in toxic and side effects and not as primary target. This approach, time and concentration to exposure of determined compound, should be standardized to have consensus in this kind of study.

\section{Conclusion}

To our experience the application of RDA brings, for the first time in M. tuberculosis, identification of differentially expressed gene, which may be related to the cell wall biosynthesis. It showed directions to study related genes in some metabolic pathways of SCAR1 and could help in studies with other anti-TB drugs with mechanism of action not totally characterized. Taken together, RDA and real-time PCR data highlight the idea that one of the SCAR1 action mechanisms may be related to the cell wall biosynthesis considering the differential expression of a cell wall hydrolase compared with the expression of $M$. tuberculosis growth not exposed to the SCAR1 and $\mathrm{INH}$. We assume that this finding, which showed high level of cell wall hydrolase, warrants further investigation. Further studies are still needed for the effective characterization of transcripts and proteins involved in the interaction of SCAR1 in M. tuberculosis.

\section{Acknowledgements}

We are grateful to the CNPq (Conselho Nacional de Desenvolvimento Científico e Tecnológico) that provided the financial support for this work and CAPES (Coordenação de Aperfeiçoamento de Pessoal de Nivel Superior) for Grant and Fundação de Amparo à Pesquisa do Estado de São Paulo (FAPESP) ref. Process: 2011/11593-7 and 2009/06499-1.

\section{Conflict of Interest: None declared.}

\section{References}

[1] Ma Z., Lienhardt C., Mcllleron H., Nunn A.J., Wang X. (2010) Lancet, 375(9731), 2100-2109.

[2] WHO (2012) WHO Report 2012, Global Tuberculosis Control, WHO Press, Geneva.

[3] Pavan F.R., Sato D.N., Higuchi C.T., Santos A.C.B., Vilegas W., Leite C.Q.F. (2009) Rev. Brasileira Farmacog., 19(1), 204216.

[4] Gupta R., Thakur B., Singh P., Singh H.B., Sharma V.D., Katoch V.M., Chauhan S.V. (2010) Indian J. Med. Res., 131, 809-813.

[5] Luna-Herrera J., Reddy M.R., Daneluzzi D., Gangadharam P.R.J. (1995) Antimicrob. Agents Chemother., 39(12), 26922695.

[6] Bergmann J.S., Woods G.L. (1998) Int. J. Tuberc. Lung Dis., 2 (8), 621-626.

[7] Gandhi N.R., Nunn P., Dheda K., Schaaf H.S., Zignol M., Soolingen D.V., Jensen P., Bayona J. (2010) Lancet, 375 (9728), 1830-43.

[8] Kumar K.N., Ramesh R., Liu Y. (2006) J. Inorg. Biochem., 100 (1), 18-26.

[9] Nascimento F.B., Poelhsitz G.V., Pavan F.R., Sato D.N., Leite C.Q.F., Selistre-de-Araújo H.S., Ellena J., Castellano E.E., Deflon V.M., Batista A.A. (2008) J. Inorg. Biochem., 102(9), 1783-1789.

[10]Pavan F.R., Poelhsitz G.V., Nascimento F.B., Leite S.R., Batista A.A., Deflon V.M., Sato D.N., Franzblau S.G., Leite C.Q. (2010) Eur. J. Med. Chem., 45(2), 598-601.

[11]Pavan F.R., Poelhsitz G.V., Barbosa M.I., Leite S.R., Batista A.A., Ellena J., Sato L.S., Franzblau S.G., Moreno V., Gambino D., Leite C.Q. (2011) Eur. J. Med. Chem., 46(10), 5099-5107.

[12]Allardyce C.S., Dyson P., Ellis D.J., Saltera P.A., Scopelliti R. (2003) J. Organomet. Chem., 668(1-2), 35-42.

[13]Pastorian K., Hawell L., Byus C.V. (2000) Anal. Biochem., 283 (1), 89-98.

[14]Palomino J.C., Martin A., Camacho M., Guerra H., Swings J., Portaels F. (2002) Antimicrob. Agents Chemother., 46(8), 27202722.

[15]Dutra V., Nakazato L., Broetto L., Schrank I.S., Vainstein M.H., Schrank A. (2004) Res. Microbiol., 155(4), 245-251.

[16]Sambrook J., Russell D.W. (2001) Molecular Cloning: A Laboratory Manual.

[17]Huang X., Madan A. (1999) Genome Res., 9(9), 868-877.

[18]Altschul S.F., Madden T.L., Schaffer A.A., Zhang J., Zhang Z., Miller W., Lipman D.J. (1997) Nucleic Acids Res., 25(17), 33893402.

[19]Livak K.J., Schmittgen T.D. (2001) Methods, 25(4), 402-408.

[20]Sholto-Douglas-Vernon C., Sandy J., Victor T.C., Sim E., Helden P.D. (2005) J. Med. Microbiol., 54, 1189-1197.

[21]Allen N.L., Hilton A.C., Betts R., Penn C.W. (2001) FEMS Microbiol. Lett., 197(2), 195-201.

[22]Bowler L.D., Hubank M., Spratt B.G. (1999) Microbiology, 145 (12), 3529-3537. 
[23]Haiser H.J., Yousef M.R., Elliot M.A. (2009) J. Bacteriol., 191 (21), 6501-6512.

[24]Chauhan A., Lofton H., Maloney E., Moore J., Fol M., Madiraju M.V.V.S., Rajagopalan M. (2006) Mol. Microbiol., 62(1), 132147.

[25]Hett E.C., Chao M.C., Deng L.L., Rubin E.J. (2008) PLoS Pathog, 4(2), e1000001.

[26]Zhang Y. (2005) Ann. Rev. Pharmacol. Toxicol., 45, 529-564.

[27]Cardoso R.F., Cooksey R.C., Morlock G.P., Barco P., Cecon L., Forestiero F., Leite C.Q.F., Sato D.N., Shikama M.L., Mamizuka E.M., Hirata R.D.C., Hirata M.H. (2004) Antimicrob. Agents Chemother., 48(9), 3373-3381.

[28]Wilson M., DeRisi J., Kristensen H.H., Imboden P., Rane S., Brown P.O., Schoolnik G.K. (1999) Proc. Natl. Acad. Sci. USA, 96(22), 12833-12838.

[29]Betts J.C., McLaren A., Lennon M.G., Kelly F.M., Lukey P.T., Blakemore S.J., Duncan K. (2003) Antimicrob Agents Chemother., 47(9), 2903-2913.

[30]Waddell S.J., Stabler R.A., Laing K., Kremer L., Reynolds R.C., Besra G.S. (2004) Tuberculosis, 84(3-4), 263-74.

[31]Karakousis P.C., Williams E.P., Bishai W. (2008) J. Antimicrob. Chemother., 61(2), 323-331. 